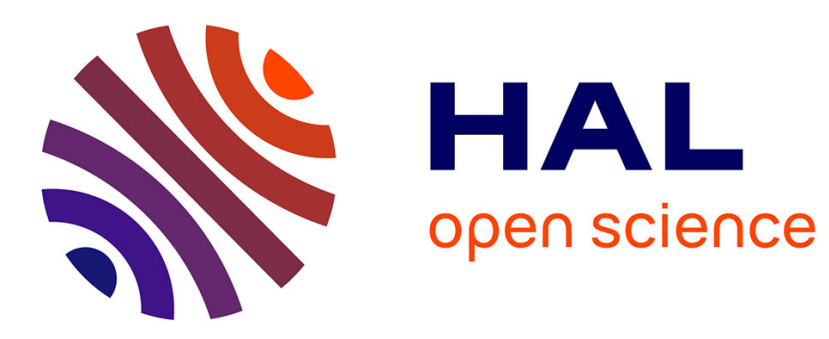

\title{
Manding Ajami samples: Mandinka and Bamana
}

Valentin Vydrin, Gérard Dumestre

\section{To cite this version:}

Valentin Vydrin, Gérard Dumestre. Manding Ajami samples: Mandinka and Bamana. Meikal Mumin, Kees Versteegh. The Arabic script in Africa: Studies in the use of a writing system, Brill, pp.225-260, 2014, Studies in Semitic languages and linguistics, 978-90-04-25679-8. halshs-00948996

\section{HAL Id: halshs-00948996 https://shs.hal.science/halshs-00948996}

Submitted on 26 Feb 2014

HAL is a multi-disciplinary open access archive for the deposit and dissemination of scientific research documents, whether they are published or not. The documents may come from teaching and research institutions in France or abroad, or from public or private research centers.
L'archive ouverte pluridisciplinaire $\mathbf{H A L}$, est destinée au dépôt et à la diffusion de documents scientifiques de niveau recherche, publiés ou non, émanant des établissements d'enseignement et de recherche français ou étrangers, des laboratoires publics ou privés. 
Manding Ajami samples: Mandinka and Bamana ${ }^{1}$

Valentin Vydrin, INALCO, CNRS-LLACAN

Gérard Dumestre, INALCO, CNRS-LLACAN

\section{Introduction}

The Manding Ajami writing tradition is much less mysterious today than it was thirty or forty years ago, when scholarly literature would settle for its passing mentions and practically no single authentic text was available. However, the number of texts published still remains extremely low, and they reflect poorly on the geographical spread of this writing system. We are still at the stage where every new text introduced into scholarly circulation is a discovery: with a high degree of probability, it represents either a new variety of the writing, a new literary genre, or even establishes the use in a new geographical area where the existence of Ajami was hitherto unknown. The texts presented here exemplify this situation.

In the following, texts of the manuscripts will be represented in four columns: the source texts in Manding Ajami, i.e. Arabic-based script orthography, a Roman script transliteration, a Roman script standard transcription, and an English translation.

In the transliteration, we tried our best to remain true to the Ajami text. We had to interpret spellings in order to segment texts into words,

\footnotetext{
${ }^{1}$ The authors would like to thank Charles Riley who volunteered to correct the English texts.
} 
whenever Arabic script (based) graphemes are written disconnected to the left. This applies to the letters 'alif, dāl, dِāl, rā', zāy, wāw, which are always spelt disconnected to the following letters in Arabic script. Therefore, it is most often impossible to judge whether a word boundary was intended by the author of the manuscript after such a letter or not. In the standard transcription, Bamana text is represented following the official orthography of Bamana language, while dialectal forms are being eliminated ${ }^{2}$. We suppose that they can be easily restored from the transliteration. Although tones are not marked in Arabic based script, lexical tones are marked in the standard transcription, while contextual and grammatical tonal changes (among them, the tonal article ${ }^{3}$ ) are ignored.

The Mandinka document is a sample of a magical text. Unlike all the other authentic documents published so far, it is authored, most probably, by an ordinary Muslim, rather than a Muslim cleric, for hunt remains a domain far remote from the sphere of pious Marabouts. 'Allāh and Muhammad are nevertheless mentioned in these texts, and Arabic words are inserted here and there for possibly extra effectiveness, such as e.g. tamāli $(3: 3,3: 4)^{4}$, and probably also qalaqa $(2: 2)$. In essence though, the religious core of this text is quite animist.

\footnotetext{
${ }^{2}$ The Ajami writing reflects a dialectal form, different from the Standard Bamana one.

3 In Bamana, a definite article is represented by a floating low tone following a noun or a noun group. In current transcription practice, it is rarely marked even in texts with tonal notation.

4 In referencing to the texts, the first number (preceding the colon) represents the number of the text, and the second one (following the colon) represents the line.

For the Bamana two-page texts, the figure preceding a slash represents the number of the text, while the second figure (following the slash) represents the page number, and the third figure, following the colon, represents the line.
} 
The other set of texts is the very first sample of the authentic Bamana writing tradition published. ${ }^{5}$ They come from San, an ancient commercial center in the southwestern part of Mali, and might represent the earliest piece ever of authentic Bamana literature.

In the following, the texts are reproduced in digitalized form, provided with an exact Roman script transliteration, a normalized transcription (with tones in Mandinka texts following Creissels et al. 1982), and an English translation including comments. Each text is followed by an analysis of the peculiarities of the Ajami variety.

\section{Mandinka hunter's incantations.}

The incantations were shown to Valentin Vydrin by Abdulay Daafee, who was his host in the Samakun village (Sédhiou region, Senegal) in October 2007. His oral comments were very helpful in decoding and transliterating of the texts. However, certain passages (given in italics in the Roman transcription) remain obscure, and their respective transliterations and translations therefore remain tentative. In particular, personal pronouns of second singular $i$ and third plural $i$ differ only in tone, and the context is usually insufficient to opt for one or the other interpretation. In such cases, decisions were taken more or less at random.

Each incantation begins with an Islamic preamble, followed by magic formulae, which sometimes consist of words which are non-existent in

\footnotetext{
${ }^{5}$ The only Bamana texts published so far (Vydrine 1998) were written at the request of Valentin Vydrin. Therefore, they may be considered as evidence for the study of the orthographic tradition of Bamana Ajami, but they do not exemplify its genres.
} 
Mandinka language (at least, they are absent from Mandinka dictionaries and are not recognized by Mandinka speakers as true words). At the end, the objects and substances are mentioned (apparently, those which are necessary for the magic action), followed by the Arabic word اسمد 'ismuhu 'his name'.

Text 1

\begin{tabular}{|c|c|c|c|}
\hline & $\begin{array}{l}\text { Transliteration } \\
\text { bsmi 'llh }\end{array}$ & $\begin{array}{l}\text { Transcription } \\
\text { 1. Bismi llahi }\end{array}$ & $\begin{array}{l}\text { Translation } \\
1 \text {. By the name of God }\end{array}$ \\
\hline & sala'ali & 2. Sala@a & 2. Greetings to \\
\hline مُدَ & muḥam²adi & Muhammadi wa & Mohammed and the \\
\hline & wa'la'liy $_{1}$ & $\begin{array}{l}\text { l'Sali Muhammadi . í } \\
\text { naril }\end{array}$ & Highest Mohammed. \\
\hline & $\begin{array}{l}\text { šubu nari minan } \\
\text { kay }_{2} \text { a kutu tu }{ }^{n} b u \\
\text { bi' 'i kun }^{n} \text { tu }\end{array}$ & $\begin{array}{l}\text { 3. Sùboo nari } \\
\text { Mìnankaña kòto } \\
\text { túnbun bé í kùntu }\end{array}$ & $\begin{array}{l}\text { 3. Animal, stop! Old } \\
\text { male antelope, ruins } \\
\text { will cut you (?). }\end{array}$ \\
\hline 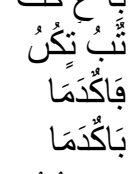 & $\begin{array}{l}t u^{n} b u \quad t i^{n} k^{n} n_{1} u \\
f_{1} a^{\prime} k u^{n} \text { dama' } \\
\text { ba'kun dama' }\end{array}$ & $\begin{array}{l}\text { 4. túnbun t'í kùnun, } \\
\text { faakundamaa } \\
\text { baakundamaa. }\end{array}$ & $\begin{array}{l}\text { 4. Ruins won't swallow } \\
\text { you, faakundamaa, } \\
\text { baakundamaa. }\end{array}$ \\
\hline & 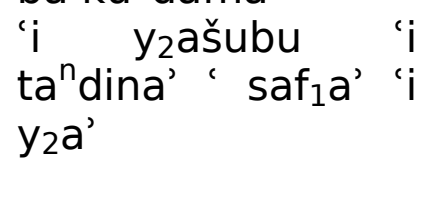 & $\begin{array}{l}\text { 5. í ñáa sùboo, ì } \\
\text { tàa, díina (jínna?), ì } \\
\text { s'à fàa í ñáa. }\end{array}$ & $\begin{array}{l}\text { 5. Animal is before your } \\
\text { eyes, take them, bush } \\
\text { spirit, they will kill it } \\
\text { before you. }\end{array}$ \\
\hline & $\begin{array}{l}\text { šutu kiy itadina' ? } \\
\text { saf }_{1} a^{\prime} \text { ' } i \text { y } y_{2} a^{\prime}\end{array}$ & $\begin{array}{l}\text { 6. Sùboo kèeñeeta, } \\
\text { díina (jínna?), ń s 'à } \\
\text { fàa í ñáa. }\end{array}$ & $\begin{array}{l}\text { 6. The animal has fallen } \\
\text { asleep, bush spirit, I will } \\
\text { kill it before you. }\end{array}$ \\
\hline & $\begin{array}{l}\text { šubu musu 'itan di ? } \\
\text { na' ? } \text { saf }_{1} a^{\prime} \text { tt }\end{array}$ & $\begin{array}{l}\text { 7. Sùbu musoo } \\
\text { yitandi ń ná, ń s 'à } \\
\text { fàa tata }\end{array}$ & $\begin{array}{l}\text { 7. Show me a female } \\
\text { animal, l'll kill it. }\end{array}$ \\
\hline تَنَايُوْ عَنِ & $\begin{array}{l}\text { ti'yuw 'ani }{ }^{\mathrm{n}} \min ^{\mathrm{n}} \text {, } \\
\text { kaya tiyuw } \mathrm{ni}^{\mathrm{n}} \\
\text { bunba sulu }\end{array}$ & $\begin{array}{l}8 . \quad \text { Tío ànín } \\
\text { mìnankaña, tío nín } \\
\text { bumban súloo }\end{array}$ & $\begin{array}{l}\text { 8. Hair and male } \\
\text { antelope, hair and the } \\
\text { root of bumban. }\end{array}$ \\
\hline 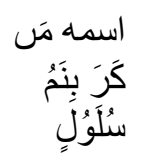 & $\begin{array}{l}\text { 'smh } \operatorname{man}_{1} \text { kara } \\
\text { binamu sulawulin }\end{array}$ & $\begin{array}{l}\text { 9. Ismahu mànkara } \\
\text { bína mú sùla wùley. }\end{array}$ & $\begin{array}{l}\text { 9. Its name is the horn } \\
\text { of the mànkara } \\
\text { antelope, red monkey. }\end{array}$ \\
\hline
\end{tabular}

Comments 
Line 1. The first spelling could be read as خسبِ | | reading hardly makes any sense. Most probably, it is an ordinary basmala (بbsm>) written in a fanciful way.

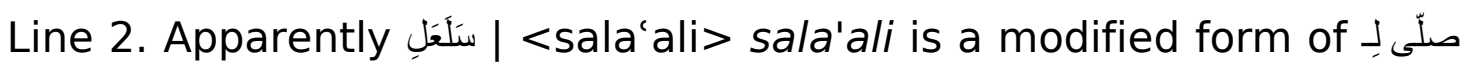
$\mid\left\langle\left. s\right|^{2} y_{1}\right|-i>$ which might be an abbreviated version of the Arabic language formula șalla 'Allah 'alayhu wa sallam 'May God bless him and grant him peace'. In the second and third incantations, it is spelled as | 1 salali> .

The word nari is absent from all available Mandinka dictionaries. The translation 'stop!' was suggested by Abdulay Daafee.

Line 3. Koto may be also interpreted as another word for 'male' (it usually appears in compounds bàakoto 'he-goat', sàakoto 'ram').

Line 4. Faakundamaa, baakundamaa are ideophones depicting the antelope's walk.

Lines 5, 6. jínna 'bush spirit' is a reading suggested by Abdulay Daafee. The literal reading díina 'religion' does not fit the context.

Line 6. kèeñee is a word from the respectful vocabulary ${ }^{6}$ for 'to sleep'.

Line 7. The final element of the line is transcribed here as tata. In fact, it might be a decorative element indicating the end of the magic formula. Cf. a similar element at the end of the San Bamana text 5.

Lines 8 and 9. It seems to be an enumeration of ingredients for the fabrication of a fetish or amulet. It looks like they were written post

\footnotetext{
${ }^{6}$ A special register used for expressing respect. For more information, see Vydrin (2008).
} 
factum, in the blank space, and line 9 was fitted into the left margin of the page.

Line 9. mànkara or mànkari is an unidentified species of antelope.

Text 2

\begin{tabular}{|c|c|c|c|}
\hline & & otion & \\
\hline 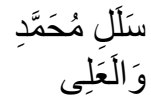 & ham & $\begin{array}{l}\text { Salali } \\
\text { wa-l- }\end{array}$ & tings \\
\hline & & Salii Muhammadi. & est \\
\hline & & & Jimbin \\
\hline & $1 a g^{n} i^{n} i^{n}$ & kalaka & kalaka jimbin. \\
\hline & $\begin{array}{l}\operatorname{mina}^{n} \text { kaya } \\
{\text { ğin } b i^{n}}^{n} \text { niti'ala }\end{array}$ & $\begin{array}{l}\text { 3. Mìnan kaña kòto } \\
\text { jinbin n'í té à lá }\end{array}$ & \\
\hline 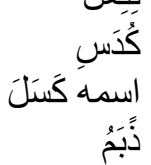 & $\begin{array}{l}\mathrm{i} \text { 'smh k } \\
\mathrm{mu}\end{array}$ & $\begin{array}{l}4 . \quad \mathrm{k} \\
\text { kásal }\end{array}$ & asalaa \\
\hline
\end{tabular}

Comments

Lines 1-2. Jimbin kalaka kalaka jimbin is a magic formula. There is a Mandinka word jimbi 'a headgear with fringes', but it hardly fits this context. Kalaka is not a Mandinka word, but might possibly be related to qalaq 'anxiety, insomnia (Arabic)'.

Line 4. Kùdasi is a verb meaning 'to remain underdeveloped', 'to fail to reach normal size' (absent from Creissles et al. 1982).

Kásalaa is an unidentified type of grass, also known as kàcancároo.

Abdulay Daafee commented: Í s'à buru jàn '(After the incantation is pronounced,) you should rub it'.

Text 3 


\begin{tabular}{|c|c|c|c|}
\hline \multicolumn{4}{|c|}{ Transcription } \\
\hline & & Salali & \\
\hline & aiy $^{1}$ & Muhammadi & Mohammed and \\
\hline 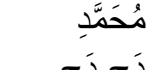 & & Sali: Muhammadi. & Highest Mohammed. \\
\hline 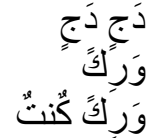 & $\begin{array}{l}\text { dağ } i^{n} \text { dağ } i^{n} \text { warik } \\
\text { warika }^{n} \mathrm{ku}^{n} \mathrm{tu}^{\mathrm{n}}\end{array}$ & $\begin{array}{lr}2 . \quad \text { Dajin } & \text { dajin } \\
\text { warikan } & \text { warikan } \\
\text { kuntun. } & \end{array}$ & $\begin{array}{l}\text { 2. Cut dajin dajin } \\
\text { warikan warikan, }\end{array}$ \\
\hline 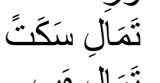 & tama'li sakata ${ }^{n}$ & 3. tamaali sákatùn & $y s$, that is, \\
\hline 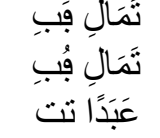 & $\begin{array}{l}\text { tama'li } f_{1} a b i \\
\text { tama'li }^{\text {'abadan, tt }} \quad f_{1} \text { ubi }\end{array}$ & $\begin{array}{l}\text { 4. Tamaali fó bìi } \\
\text { ábaday. }\end{array}$ & $\begin{array}{l}\text { 4. Always, up to th } \\
\text { day, forever. }\end{array}$ \\
\hline 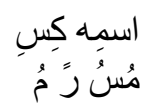 & $\begin{array}{l}\text { Psmih kisi musu ra }{ }^{n} \\
\text { mu }\end{array}$ & $\begin{array}{l}\text { 5. Ismihu kèse } \\
\text { mósoran mú. }\end{array}$ & $\begin{array}{l}\text { 5. Its name is a ruk } \\
\text { bullet. }\end{array}$ \\
\hline
\end{tabular}

Comments

Line 2. dajin dajin warikan warikan are magic formulae - These are not Mandinka words.

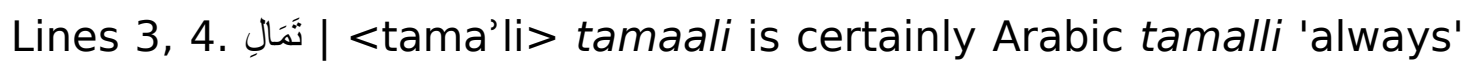
(this word is absent from the everyday Mandinka).

Line 3. نsakatan> sakatan was read by Abdulay Daafee as sókotùn 'that is'. Cf. in Creissels et al. 1982 sàko, sòko 'surtout, encore plus, à plus fort raison'.

Line 5. Mósoran is an instrument name from the verb móso 'to rub while spitting and pronouncing incantations'. This line denotes the destination of the incantation.

3. Grammatological remarks to the Mandinka hunters' incantations 
On the grounds of the spelling of the palatal nasal sonant $n^{7}$, these texts would belong to the main Mandinka Ajami variety (cf. Vydrin, this volume): $n<\tilde{n}>$ is $O K$ represented by . Further grammatological characteristics of this document are:

- $\quad-s$ is usually written as $\sin$, except for one case where it is written as šĩn, i.e. شُبُ | Š̌ubu> sùbu 'animal, meat'.

- $\quad-j$ (a voiced palatal affricate) is spelled as eiher $\dot{j}$ or $\underset{ }{ }$, with the latter appearing both word-initially and word-finally ${ }^{8}$.

- $\varepsilon$ is used as support for any diacritic expressing a vowel, when it is not preceded by a consonant. Furthermore, it also is used for spelling of the palatal oral sonant $y$, although there is only one occurence in the text, i.e. 1 < 1 'itan di> yitandi 1:7 'to show'.

- The nasal syllabic phoneme $\eta$ (which appears in Mandinka in only two words: $\eta^{\prime}$ 'I', ’̀ 'we') is written as hamza, i.e. - (Which could also be an (ain, i.e. ع) without any support or vocalic diacritics.

- $\quad$ Letters representing emphatics in the orthography of Arabic are not used, with the only exception of the rather obscure word فَ $<q_{1} \operatorname{alaq}_{1} a>(2: 2)$.

- $\quad$ Nūn, qāf and fă’ are written in the Maǵribī way: In word-final positions, nūn has normally (with only one exception) no dot, while qāf is always spelled with one dot above, i.e. ف, and fấ' always with one dot below, i.e. 4 .

\footnotetext{
${ }^{7}$ In the text, there is not a single word containing a $p$, which makes the other diagnostic criterion (see Vydrin, present volume) inapplicable.

${ }^{8}$ In one word $j$ is probably written using $d \bar{a} l$, L $\mid$ | < dina'> (incantation 1, lines 5 and 6). However, we are not sure about the interpretation of this word.
} 


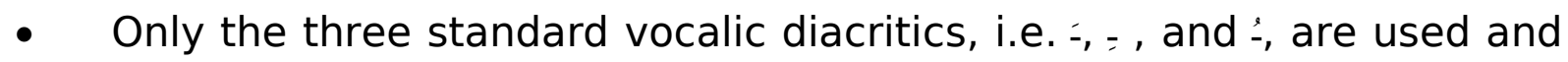
there are no attempts to differentiate closed and mid vowels.

- 'Alif, wāw, and yā' do not indicate in any way length of vowels. These letters appear mainly at the end of words, sometimes in disagreement with the preceding vocalic diacritic, and always without any visible correlation to vowel length, e.g. Цپ bé 'imperfective marker'. However, such 'fake length' is much less frequent than in some other Mandinka Ajami texts.

- The syllable-final nasal element is usually rendered by tanwīn, e.g. | <sulwulin > sùla wùlen 'red monkey' (1:9), and rarely by nūn as

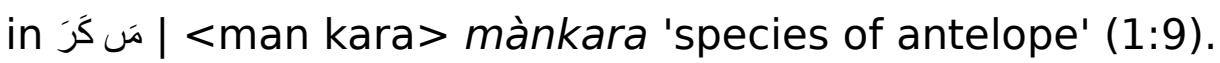

\section{Bamana texts from San}

In 1972, during one of his sojourns in Mali, Gérard Dumestre dispatched a friend of his, Almamy Malik Yattara, to the ancient commercial town of San to look for old Bamana manuscripts. Almamy Yattara was a remarkable representative of the Muslim intelligentsia of Mali, and two books were dedicated to him (Yattara \& Salvaing 2000; 2003). Being a Muslim cleric, it was much easier for him than for a white man to gain confidence among his coreligionists. A couple of weeks later, Almamy Yattara returned to Bamako with copies of five manuscripts: Bory Bary, the head of one of the Islamic families of San, allowed him (for a modest recompense) to copy by hand texts, which had originally been written by Bary's father, Amadu Bary, in 1911. 
Being literate in French, Almamy Yattara provided each manuscript with a brief comment, which yet proved difficult to understand at times. These comments are reproduced here in an English translation, while the French originals are given in footnotes in the author's own orthography. There is also a comment written by Almamy Yattara referring to the entire set of the documents:

The length of this sheet is equal to the origin. I have written them from a white paper, the characters are equal.

The gift for these five documents was 6000 Malian Francs, for I gave 3000 Francs for two initial ones, when I saw that I was too tough, and I did it in order to have more, if any. And for the other three manuscripts I gave 3000 Franc. It is the time of working the farm. Nobody has time. By Almamy Yattara. ${ }^{9}$

Today, 39 years after the manuscripts were copied by Almamy Yattara and exactly 100 years after they were created, we proceed to their publication.

\section{Text 1}

Almamy Yattara's comment:

\footnotetext{
${ }^{9}$ The original text:

les longere de sette feille et egal de loriginal. je les estrié de papié Bilansse le karactere et egalla

le cado de cet 5 dockimen. eté 6000 faran. pissique les 2 prumier je done $3000 \mathrm{~F}$ lorssque jevi jessi tro diure et je fai sa pour avar encor si yana. et avec le 3 maniscri- lotre je done $3000 \mathrm{~F}$. ya le travaus de samnp. toule monde nonpa de tan. par Almamy Yattara
} 
The possessor of the document "Karantela Kolomba, the sacred well of San", Bory Bary. Written by his father in Arabic characters in San for him in 1911. The copyist Almamy Malik Yattara in San in $1972 .{ }^{10}$

Page 1

Transliteration

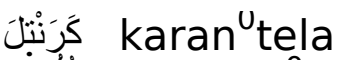

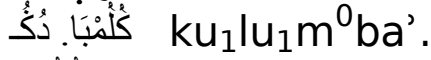

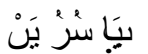

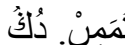

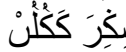

سُرْ يَنْ

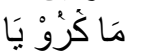

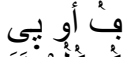

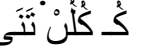

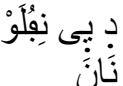

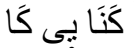

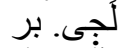

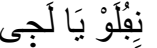

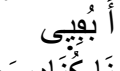

نا كنادِيرِي

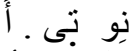

تذغ بسَّانَاً

مَنسان

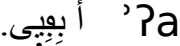
karan ${ }^{0}$ tela ku $u_{1} u_{1} \mathrm{~m}^{0} \mathrm{ba}_{1}$ $t_{1} l u_{1} n^{0} k-e$ bik-e '? da la

$\operatorname{san}^{U} \mathrm{wu}_{1} \mathrm{wu}_{1}$

nimurnik-e

seray $_{1}$ maw $^{0}$ bita'

mu $u_{1}$ ik-e

$\mathrm{ku}_{1} \mathrm{w}$ la. ku$u_{1} \mathrm{w} \cdot \mathrm{miyn}_{1}{ }^{u}$

$\mathrm{tu}_{1} \mathrm{k}_{4}-\mathrm{u}_{1}$

san $^{0}$ keyrey $_{1}$

nitulu ${ }_{1}{ }^{U}$ k-e mak-e '?a

dala ǧek $k_{4}-e$ tesu $r_{1} \mathrm{ru}_{1}$

'Pu bif ${ }_{1} \mathrm{u}_{1} \mathrm{lu}_{1}$

katulu $_{1} \mathrm{nk}_{4}-\mathrm{e}$
Transcription

$1 . \quad$ Karantela

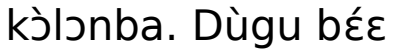
y'à sòro yàn

2. tùma mín dùgu sìgira kà kذ̀lon sj̀ro yàn.

3. Màa k j̀row y'à fó ánw yé kó kذ̀lon tàna dè yé ní fúlaw nàna,

4. ù kàn 'à yé $k$ 'à lájé. Bàri ní fúlaw y'à lájé à b'ù fíyen

5.

n'à 5. if they are lucky, kùnnadíyara, n'ò té, à tìgi b ć sà. Ní à má sà,

6. à $b$ ह́ fíyen.

Karantela

kذ̀lonba, túlonke

bé ké à dálá

7. sàn ó sàn. Ní

mónniketuma

séra, màaw $b$ ह́ táa mónni ké

8. kذ̀ lá. Kذ̀ mín

tóg

Sankeeree.

kó

9. Ní túlonk $\varepsilon$ má

ké à dálá, jége té sว̀ro. Ù bé fólว

key $_{1}$ 10. kà túlonk $\varepsilon$ ké
Translation

1. Big well of Karantela. When the entire village found it here,

2. when the village was founded the well was here.

3. Old people told us that the taboo of the well is: if Fulbe come,

4. they should not see it. But if Fulbe see it, it will blind them, otherwise, they will die. If he does not die,

6. he will become blind. Plays are organized near the big well Karantela 7. every year. When the time of fishing comes, people go to fish

8. to the river. The river whose name is Sankere.

9. If games are not played near it, there will be no fish. First 10. they play near

\footnotetext{
${ }^{10}$ The original text:
}

le détentéer de docimen Karantéla Kolomba le piu sacré de- san. Bory Bary ecri par son pere an karactere- arabe a san pour loui, en 1911 a san. le copisste Almamy malick Yattara a san en 1972. 


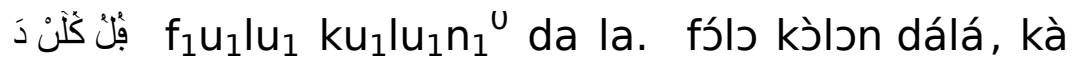

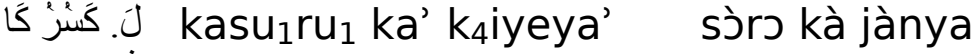

كينيان

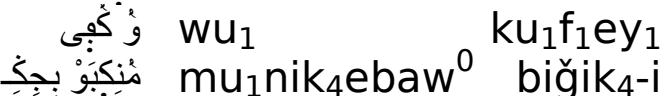
نَ

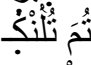

منْكيرَ بن

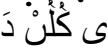

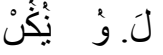

s

سَنْكِير لَ.

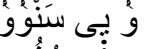

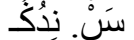

مَا بِلَ سُعَىَى

"نينى

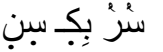

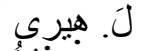

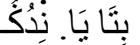

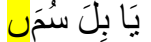

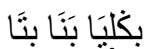

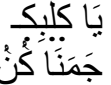

مَسَّاوْ

بسنَا دِمِسَّوْ

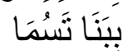

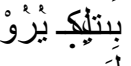

Page 2

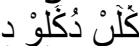

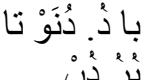

du $u_{1}$ dunaw ${ }^{0}$ $\mathrm{yu}_{1} \mathrm{ru}_{1} \mathrm{du}_{1} \mathrm{n}_{1}{ }^{0}$

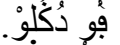

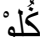

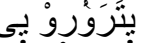

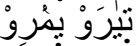

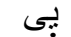

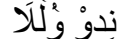

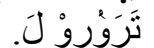

بنَانَابينين

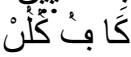

苗

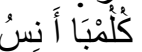

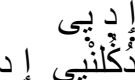

san keyre la wu tuma

tulu $n^{0} \mathrm{k}_{4}$-e

min keyra yen ${ }^{0}$ 'Py

$\mathrm{ku}_{1} l u_{1} n_{1}{ }^{0}$ da la. $\mathrm{wu}_{1}$

$y_{3} u_{1} k_{4} u_{1} n_{1}{ }^{0}$

bik-e

$\mathrm{mu}_{1}$ nikey $\mathrm{yu}_{1} \mathrm{ru}_{1} \mathrm{la}$.

sankey $_{1} r$ la. wu yey

sanwu $u_{1}$ u $_{1}$

niduk $_{4}-\mathrm{u}$ ma

sumay $_{1}$ by $_{3} \mathrm{ey}_{1}$

$\operatorname{san}_{1}$.

bila

11. wò $k$ ว́fरे mónnikebaaw bé jìgin Sankeere la . Wò tùma túlonke

12. mín $\mathrm{k}$ ćra yèn à yé (?) kذ̀lon dálá. Wò nògon bé

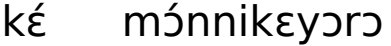
lá.

13. Sankeere la. Wò yé sàn ó sàn . Ní dùgu $m$ 'à bìla, sùman bé nè,

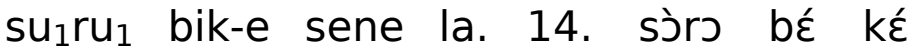
heyrey $_{1}$ bita' ya’. sène lá, hére bé niduk $_{4}-u$ ya’ bila táa ná. Ní dùgu suman $_{1-}$

bk4leya' bana' bita' ya' kelebik-e ğamana' $\mathrm{ku}_{1} \mathrm{nu}_{1}$ masa' $^{0}$

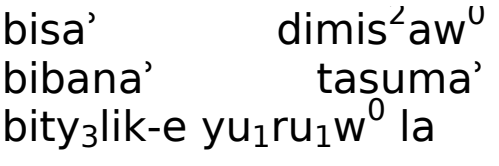
y'à bìla, sùman

15. bé gèleya, bàna b é táa ná,

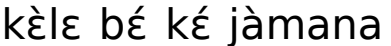
kJ́nว, màsaw

16. bé dénmisenw bé bàna, tásuma $b$ ć tíneli ké yórow lá.

$\mathrm{ku}_{1} \mathrm{lu}_{1} \mathrm{n}_{1}{ }^{u} \mathrm{duk}_{4} u \mathrm{lew}^{\mathrm{U}} \mathrm{de}$

$f_{1} u_{1} w \quad d^{2} k_{4} u l e w^{u}$ duk$_{4} u l e w^{0}$

yetarawu $_{1}$ rew $^{0} \quad$ yey $_{1}$ tey $^{a}$ raw $^{0} \quad$ yemu $_{1}$ riw $^{0}$

yey $_{1}$

nidew ${ }^{0}$ $w_{1} l u_{1} l ' a$

tarawu rew $^{0}$ la. '?u bita' na' yeyen $_{1}{ }^{0} \mathrm{ka}$ $f_{1} u_{1} k u_{1} l u_{1} n_{1}^{0}$ yey

karan 'tela kul lu m 'ba' 'Pa nisu ' ${ }_{i}$ e de yey duk4ulen ${ }^{0}$ yey $_{1}$ ' $P_{i} e$ de yey $_{1}$ dunaw

$4 . \quad$ "Karantela
1.

Kòlon, 1. Natives know the dùgulenw dè $b$ 'à well. Foreigners dón. Dúnanw t 'à don't know its yóro dón,

2. fóo dugulenw . Dùgulenw ye

Taraworew yé Teeraw yé móriw yé.

3. Ní dénw wólola Taraworew lá , ù bé táa $n$ 'à yé yèn k'à fó kذ̀lon yé kذ̀lonba, á ní sú! É dè yé dùgulen yé, é dè yé dúnanw

whereabouts, 2. only natives do. Natives are Traore, and Tera are Muslims.

3. When Traore children are born, they bring the child there and say to the well:

4. "Karantela, the Big Well, good evening! You are the native, it is you the well, then they move away

11. then the fishers descend to Sankere. At that time the game

12. that is played there ...? near the well. The same thing is done at the fishing place,

13. near Sankere. It happens every year. If the village does not abandon it (the rite), food will be abundant,

14. the harvest will be good, the peace will advance. If the village abandons it, food

15. will be scarce, diseases will get ahead, there will be a war in the country, rulers

à, 16. will die, children will fall ill, fire will wreak havoc. 


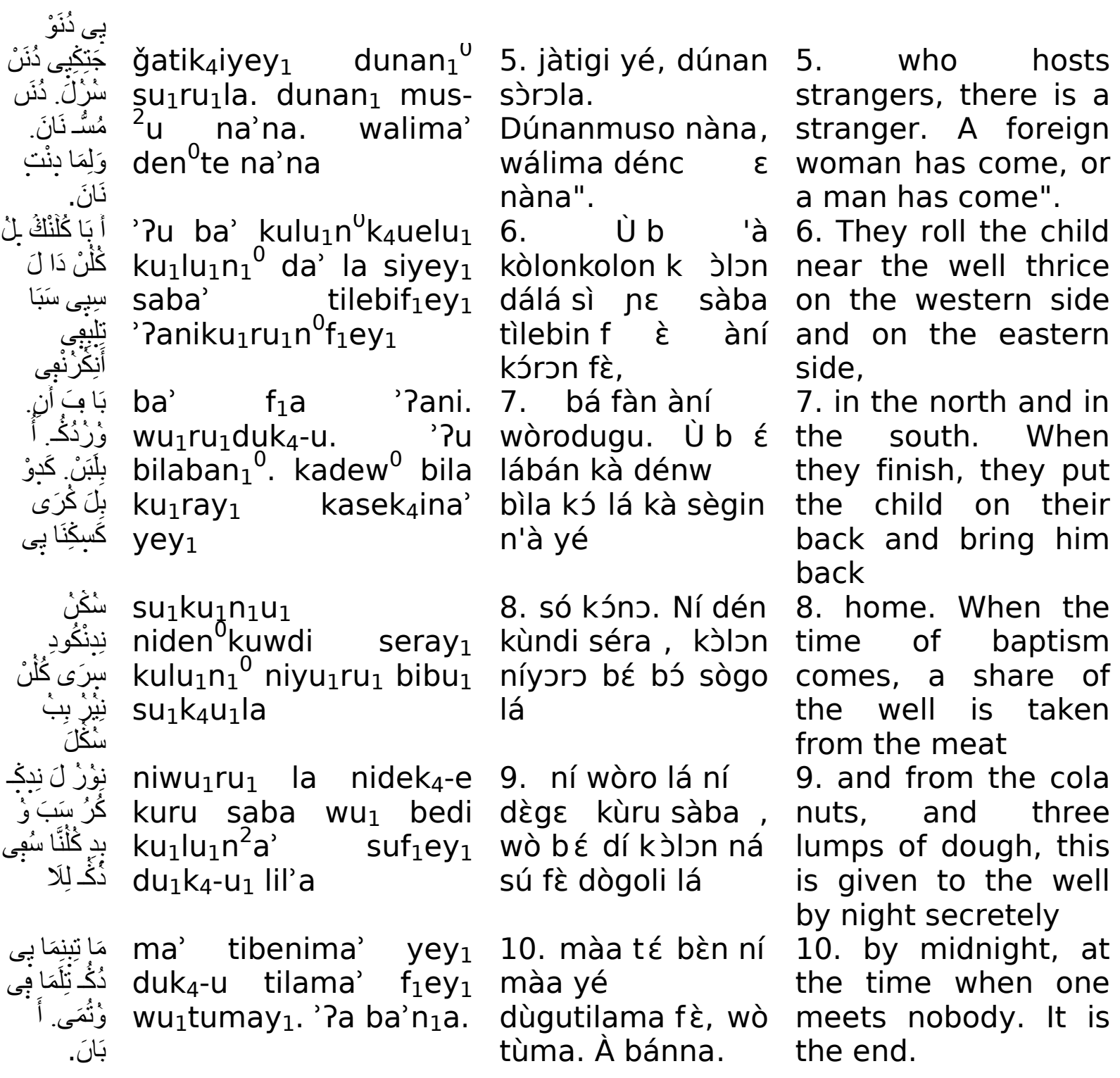

Text 2

Almamy Yattara's comment:

The possessor of the document "Janaba koli" is the same Bory Bary, inherited from his father who wrote (it) in order to teach religion to the Bamana people. The copyist Almamy Malik Yattara. ${ }^{11}$

\footnotetext{
${ }^{11}$ The original text:

le detantere de docimen de janaba koli le meme Bory Bary airite a son pere que ecri pour - enssenge les Banbara en religon. le copissite Almamy malick Yattara.
} 
Transliteration

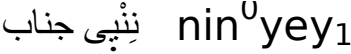

كولِيَى

جَانَابَ كَول

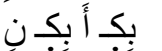

جي

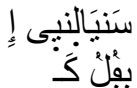

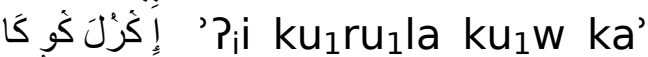

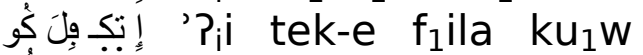

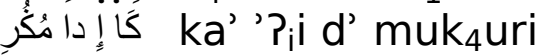

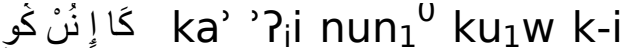

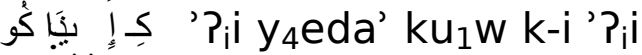

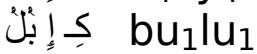

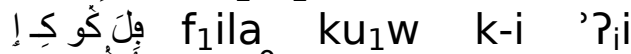

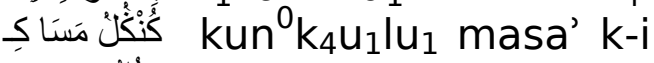

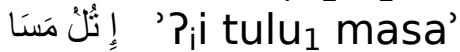

كَ

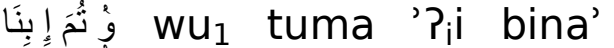

كُولِ دمِن $\mathrm{ku}_{1}$ wli dmine

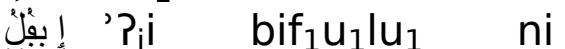

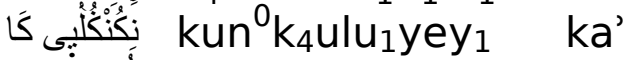

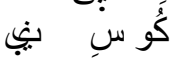

kuw siy ${ }_{4} e y_{1}$ saba

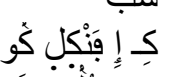

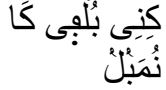

كُو كَا لَجِى

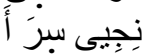

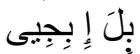
ألَّن

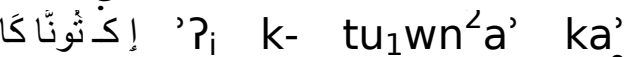

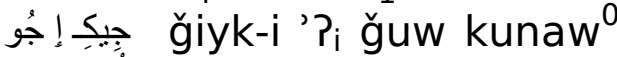

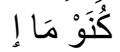

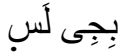

ma' '? biğiy 1 lase

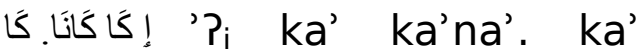

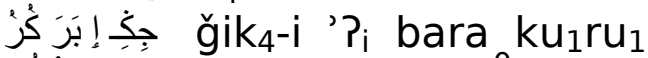

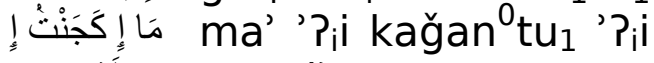

يَر yere l'a

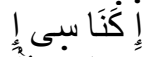

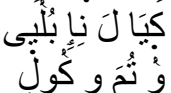

'? ${ }_{i}$ kana' sey ${ }_{1}$ ' ${ }_{j} i$ keya' la ni'?i bulu$_{1}$ yey $_{1}$ wu $_{1}$ tuma w ku$u_{1} w i$

كلب أبِوَسنَ

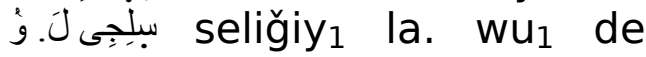

Transcription

1. Nìn yé jànaba

kòli yé.

2. Jànaba kòli $b$ ć

kź, à b $\varepsilon$ ké ní jí

sániyalen yé. Í b

fólo kà

3. í kúrola kò kà í

tége fila kò kà í dá

múguri

4. kà í nún kò k 'í

néda kò k'í bólo

5. filla kò $\mathrm{k}$

kùnkolo màsá ${ }^{12} \mathrm{k}^{\prime}$ í túlo màsá

6. k'í sèn kò . Wò tùma í b ćnà kòli dámìne.

7. Í b $\varepsilon$ fólว ní 7. Start with your kùnkolo yé $k$ 'à kò sìne sàba

8. k'í fàn kélen kò kínibolo $f$ हे, kà númabolo

9. kò k 'à láj ह́ ní jí séra à b éc lá. í bé jí lásé

10. í kòton ná kà jí ké í jùkunanw mà . í bé jí lásé

11. í kó à ká nà kà jìgin í bàrak Jro mà, í ká jàntó í yère lá

12. í kàná sé í

kèya lá ní í bólo

yé. Wò tùma wò

kòli

13. kélen, à b 'í 13. are performed,

wàsa séliji lá . Wò the ablution water
Translation

1. These are the ablutions.

2. The ablutions are performed with clean water. First of all,

3. wash your lower parts and your both hands, rinse your mouth,

4. wash your nose, wash your face, wash both of your

5. hands, scrape your head and your ears,

6. wash your feet. Then begin your ablutions.

head and wash it thrice,

8. wash one side with your right hand, whash your left

9. hand and examine if water has reached everywhere. Bring water

10. to the lower part of your back, pour water on your buttocks. Make it in a way

11. that the water may reach the lower belly, take care of

12. not touching your private parts with your hand. Then the ablutions

\footnotetext{
${ }^{12} \mathrm{~A}$ form inexistent in Standard Bamana, which might be Maninka in origin.
} 


\begin{tabular}{|c|c|c|c|}
\hline 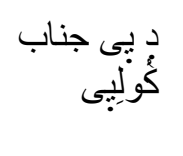 & yey $_{1}$ ğn'b ku wliyey $_{1}$ & $\begin{array}{l}\text { dè yé jànabakoli } \\
\text { yé }\end{array}$ & $\begin{array}{l}\text { has sufficed you. } \\
\text { These are the } \\
\text { religious ablutions }\end{array}$ \\
\hline 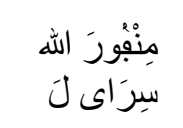 & $\begin{array}{l}\min ^{U} f_{1} u_{1} w r a \\
\text { sira'y }^{\prime} y_{1} l a\end{array}$ & $\begin{array}{l}\text { 14. mín f óra Ála } \\
\text { síra lá. }\end{array}$ & $\begin{array}{l}\text { 14. that were } \\
\text { mentioned in God's }\end{array}$ \\
\hline 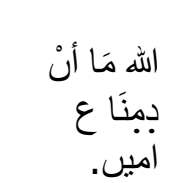 & 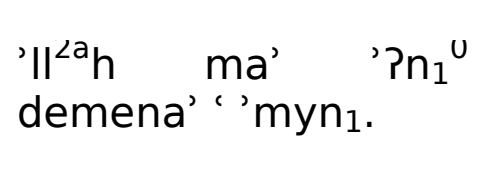 & $\begin{array}{l}\text { 15. Ála má án } \\
\text { dèmena. Àmiina. }\end{array}$ & $\begin{array}{l}\text { way. } \\
\text { 15. May Goc } \\
\text { Amen! }\end{array}$ \\
\hline
\end{tabular}

Text 3

Almamy Yattara's comment:

The possessor of the document "Kalan bidamine ni alatogo ye", Bory Bary, inherited from his father, the same Madou Bari, who wrote (it) in order to teach the Bamana people. The copyist Almamy Malik Yattara in San in $1972 .^{13}$

Page 1

Transliteration

kalan ${ }^{\cup}$ bidamine ni ' $\|^{2 a} h \quad t_{1} k_{4} u_{1} y_{e y}$ $' ? n_{1}$ salila ' $\|^{2 a} h$ tiyra la' miyn $_{1}{ }^{u} t_{1} k_{4}-u_{1} k-u_{1}$ mhmd hin ey ${ }_{1}$ nikisi bi'? k-a. 'Pa k-u sila'meya'

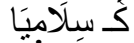

سَمَانبنْ يَى

دُورُ بُى

كَ'

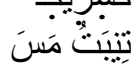
مَ

أحُو نِمَسنَ $f_{1} U_{1} W$

\section{sama'sen $_{1}{ }^{0} \quad$ yey $_{1} 3 . \quad$ sàmasen yé} duwru yey $_{1}$ kasereyak-e tiys batu masa mak-e

nimasa sèereya $k$ tìnebaats $m a ́ k \varepsilon^{14}$
Transcription

1. Kàlan bé dámìne ní Ála $\mathrm{t}$ jóg yé àní sálila Ála círa lá 2. mínt j́go kó Muhammad, híne ní kísi b é à kàn. À kj́ silameya dúuru yé : kà
Translation

1. Learning begins with the name of God and greeting to the Messenger of God 2. whose name is Muhammad, he is merciful and he is the Saviour. Besides, Islam

3. has its five buttresses: to witness

$\varepsilon$ the verity of the King màsa Lord:

4. say that Lord is

\section{${ }^{13}$ The original text:}

le detantere de locimen kalan bidamine ni alatogo ye Bory Bary airite a son pere le meme Amadou Bari que eciri pour enssenge le Bambara. le copisste - Almamy malick yattara. a san en 1972

${ }^{14}$ The syntax of this phrase is not clear. 


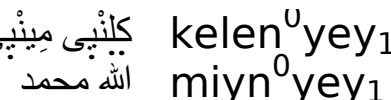

فَنَى الله كَ mḥmd f 1 anay

تِينِنْ

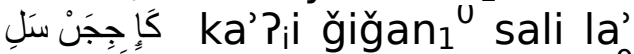

لَا لَانِ سَنِيًَا

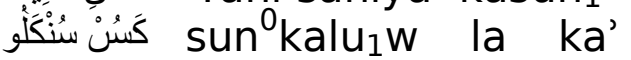

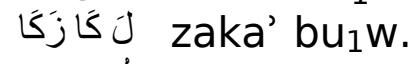

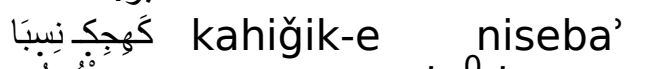

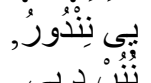

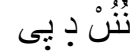

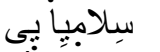

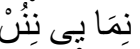

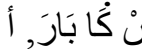

كير keyra sila'mayey.

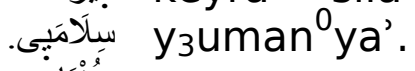

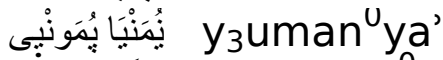

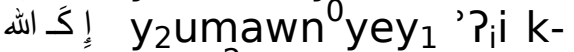

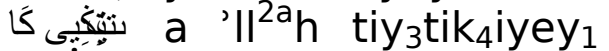

محمد تيتيكيَا

إِكَا ملكِّز

تيتنَيَّى كَا

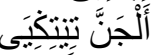

كَا جَاَُنَّنَ

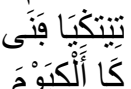

ğah²anªma

tiystk4iya' $f_{1}$ anay $_{1}$

ka' 'Pal kiyaw ${ }^{0}$ ma

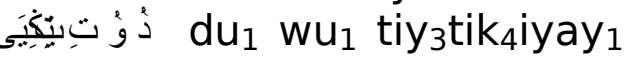

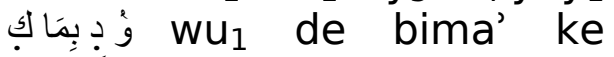

سِلَّمَا 'sila'ma'

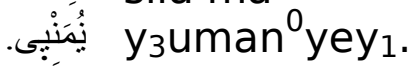

سِلَاْمَا sila'ma'

'Pabiwakali 'll<ah la'.

لَ.

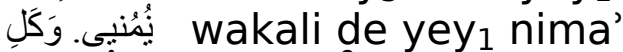

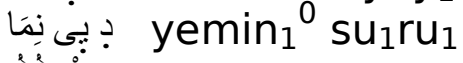

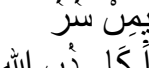

'a ka' du en ' 'll ${ }^{a} h$ de

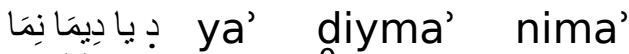

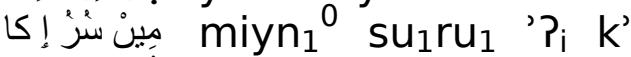

دُ الله د دمَا

دِيَتى نِاٍِ بَنَنَا

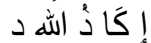

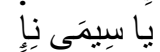

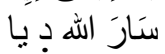

سبيمَى سمَ

diymay $_{1}$

ni' ${ }^{i} \mathrm{i}$

banana' ' $P_{i} i$ ka' du 'Ila de ya' siymay ni'?ii sa'ra 'll ${ }^{a} h$ de $y^{\prime}$ seymay $_{1}$ yé mín yé Ála

Muhamadu fána

Ála kà cíden dòn;

5. kà í jíjà sáli

àní sániya ; kà sún

súnkalo lá ; kà jáka

bó;

6. kà híji k ع́ n'í sé

b'à yé . Nìn dúuru ,

nìnnu dè yé

silameya yé.

7. Ní màa yé nìnnu

dón k'à báara, à

kéra sìlama yé

Nùmanya.

8. Nùmanya:

nùman yé í ká Ála

tìnetigiya kà

Muhammadu

tìnetigiya, í ká

mèlहkहW

9. tìnetigiya, kà àlijana tìnctigiya kà

jàhanama

tìnctigiya fána kà

álikiyama

10. dón wò

tìnetigiya, wò dè bé màa $k$ ć silame nùman yé. Silame

11. à bé wàkali Álla lá. Wàkali nùman yé. Wàkali dè yé: ní màa yé mín sj̀ro,

12. à k 'à d j́n, Ála dè y 'à d 'í mà ; n'í má mín s j̀ro, í k 'à dón, Ála dè m'à

one, and it is God, and Muhammad is the Prophet of God;

5. to be assiduous in prayer and in cleanliness; to keep Lent; to donate alms;

6. to make hadj if you can. These five (duties), it is the Islam.

7. If a person knows these (duties) and performs them, he is a Muslim. Goodness.

8. Goodness: It is good that you recognize the truth of God and Muhammad, the truth of

9. angels, the truth of Paradise, and also the truth of Hell, the truth

10. of the Doomsday, it is what makes one a good Muslim. A Muslim,

11. he relies on God. To rely, it means: whatever one receives,

12. he knows that it is God who has given it to him; whatever you do not receive, you know that it is God who

13. hasn't given it to you. If you have fallen ill, you know that it is God who has sent it to you, if you 
die, it is God who has sent it to you.

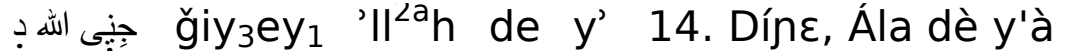

14. The world, God

يأَدَد da da

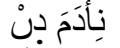

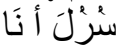

سن.

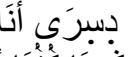

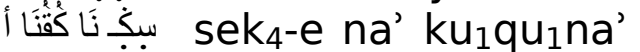

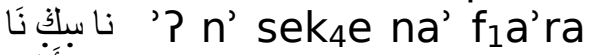

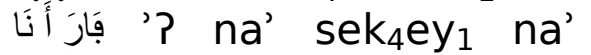

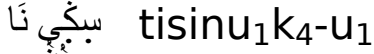

أناسبذنان '? n' sek4-e n'

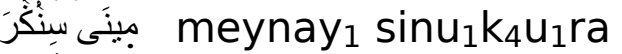

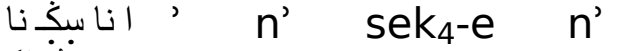

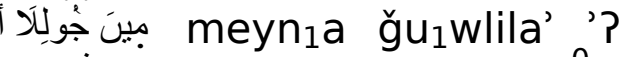

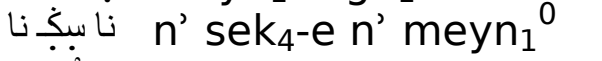

•مين

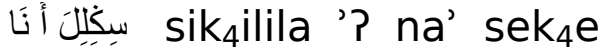

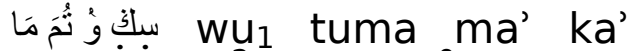

نَّ

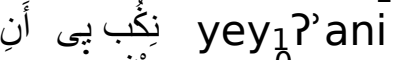

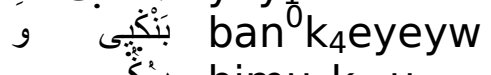

$\mathrm{bimu}_{1} \mathrm{k}_{4}-\mathrm{U}_{1}$

dá ní $\mathrm{s}$ ह̇gen yé. Ní

ádamaden s j̀rola,

à ná sègen.

15. N'à d ह́scra, à

ná s ह̀gen. N'à

kógગra, à ná s c̀gen.

N'à fàara , à ná

sègen. N'à tí

sìnogว,

16. à ná s ह̀gen. N'à

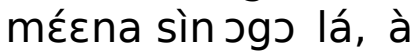
ná s ह̀gen. N'à mécnna jòli lá, à ná ડદ̀gहn. méधn(na)

N'à

17. sìgili lá, à ná sègen. Wò tùma màa ká Ála d j́n ní kó béc (?) yé, àní bánge yé (?), wò bé mògว Page 2

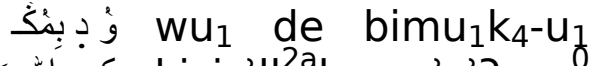

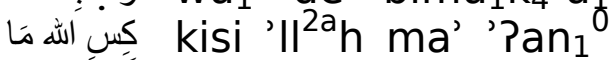
Risiray

'? أنَانَ '? ba'na
1. wò dè $b$ ź mòg kísi. Ála má án kísira.

2. À bánna. has created it with sufferings. If a human being is born, he will suffer.

15. If he fails, he will suffer. When he arrives to maturity, he will suffer. If he is killed, he will suffer. If he is not asleep,

16. he will suffer. If he sleeps too long he will suffer. If he stands too long, he will suffer. If he

17. sits too long, he will suffer. So, man should know that God is everything, he is

Creator (?), he

1. he saves man. May God save us.

2. It is the end.

Text 4

Almamy Yattara's comment:

The possessor of the medical document for hernia that is named "kookily foura". The same Bory Bary. Written by his father Amadou Bary, a Muslim cleric. The copyist Almamy Malik Yattara in 1972 in San. ${ }^{15}$

\footnotetext{
${ }^{15}$ The original text:
} 
Transliteration

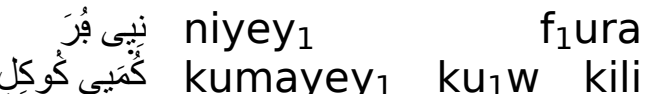

$f_{1} u r a$

$f_{1}$ ura miyn ${ }_{1}^{u}$ be $k u_{1} w$

kili $f_{1}$ ura k-e. ku $w$ kili

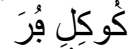

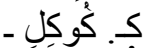

نَاَبَنَّا أبك

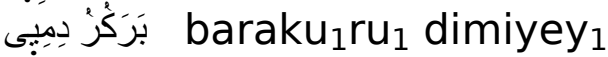

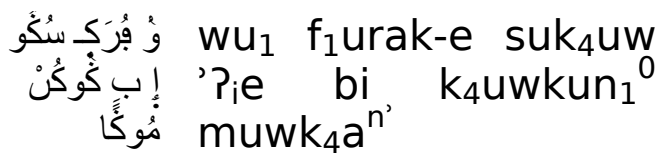

أن أن كلِ بُو إِ

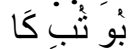

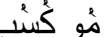

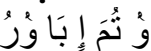

إِ

كَّذ دُونِى

كَالَ بَاكَ

تِِيَّى إِبا

سبيَيى سئكنو

كا دَّك

كَا سُوَ قَتَنى

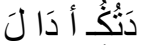

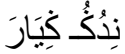

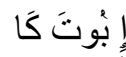

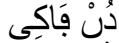

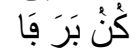

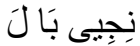

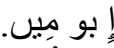

نَ

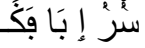

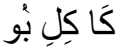

كَا دُنْ كَا

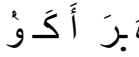

تُعَى إِ

بيجَنْن إِ

'Pani kele bu ${ }^{2}{ }^{\prime} ?_{\mathrm{i}} \mathrm{i}$ $b_{1} w$ tu $b i$ ka' $m_{1} w$ $\mathrm{ku}_{1} \mathrm{Su}_{1}$ be

$w_{1}$ tuma ' ${ }^{i} i$ ba' wu ru '? ${ }^{i} i$ bi tum ${ }^{0}$ butu $k_{1} k_{4}-u_{1} d_{1} u_{1}{ }^{2} y_{1}$

ka' la $f_{1} a^{\prime}$ k- timiyay 1 ' $\mathrm{p}_{\mathrm{i}} \mathrm{b}$ ' siyy

$\mathrm{su}_{1} \mathrm{Ku}_{1} \mathrm{nu}_{1} \mathrm{~W}$ ka'

datuk $_{4}-u$

ka' $\mathrm{su}_{1} \mathrm{w} \mathrm{f}_{1} \mathrm{anay}_{1}$ datuk $_{4}-u$ '? da' la niduk4-u k4iya'ra

' $\mathrm{P}_{\mathrm{i}} \mathrm{bu} \mathrm{bu}_{1} \mathrm{wta} k \mathrm{k}^{\mathrm{\prime}} \mathrm{dun}_{1}{ }^{u}$ $f_{1} a^{\prime} k^{\prime} y_{1} k_{1} n_{1}$ bara $f_{1} a^{\prime}$ niğiy $_{1}$ ba' la

' ${ }_{j} i$ bw miyn $n_{1}$. niyey $\mathrm{n}^{0} \mathrm{k}_{4}$ eleni $\mathrm{su}_{1} \mathrm{ru}_{1}{ }^{\prime} \mathrm{P}_{\mathrm{i}} \mathrm{i}$ ba' $f_{1} a k_{4}-a$ ka' kili $\mathrm{bu}_{1} \mathrm{w}$

$\mathrm{ka}^{\prime} \mathrm{dun}_{1}{ }^{\mathrm{k}} \mathrm{ka}$ ' haera '?

k-a $w_{1}$ tumay $_{1}$ ' $\mathrm{P}_{\mathrm{i}} \mathrm{i}$

biyğan ${ }^{0} u_{1} \mathrm{~W}^{\prime} \mathrm{P}_{\mathrm{i}} \mathrm{i}$ yerae
Transcription

1. Nìn yé fúra

kúma y é, kókili fúra.

2. Fúra mín b $\varepsilon^{2} 2$. A medicine which kókili fúrak

Kókili,

$\varepsilon$. heals

Testicles,

3. n'à bànana, à 3 . if there is a bé

bàrakırodimi yé. ké disease, it manifests itself as an ache of the lower belly.

4. Wò fúrak súgu: é b gذ̀kun ${ }^{16}$ mùgan

Translation

1. These are words of a medicine, a testicle (hernia) medicine.

4. Here is a means to $\varepsilon$ treat it: get twentyone beans of the wonderbean plant (Canavalia ensiformis),

5. àní kélen $b$ j, í 5 . boil them till they b'ò tóbi $\mathrm{k}$ 'à $\mathrm{m}$ jे are very well cooked. kóscbe.

6. Wò tùma í b 'à wóro, íb

ह́

Tumbutu kذ̀g dóวni

7. k'à lá f 'à ká

tímiya. í b'à sìi só

kóno k'à dátúgu

8. kà só fána

dátúgu à dá lá . Ní dùgu jéra

9. í b'ò tà $\mathrm{k}$ 'à dún

f'à k'í kónobara fá.

Ní jí b'à lá

10. í b 'ò mìn. N'í yé ngèlennin sòro í b'à fàga k'à kíli bó

11. k'à dún kà héra à kàn. Wò tùma í b 'í jàntó í
6. Then peel them, put a little salt of Tombuktu

7. to make it mild. Put it in the house and close it

8. and also close the door of the house. At dawn,

9. take it and eat it so that it fills your belly. If there is a juice,

10 drink it. If you can get a ground squirrel, kill it, take its testicles

11. and eat them, and be reassured with it. Then abstain

le detantere de docimen medicalman pour le hérni conapele kookili foura. le meme Bory Bary - eciri par son pere Amadou Bay marabou. le kopisste Almamy malick Yattara. a 1972 a san.

${ }^{16}$ It could be also nkòkun 'head of a species of locust', although the following context makes this interpretation less probable. 


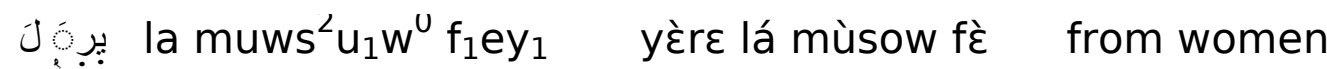

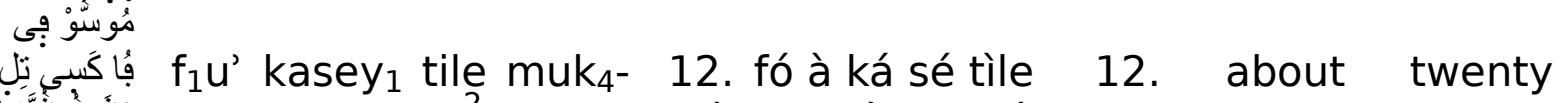

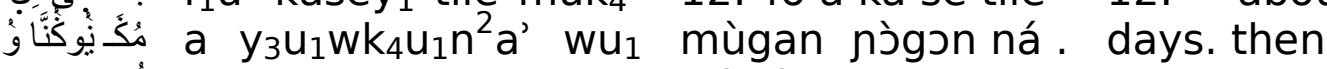

' ${ }^{\prime}$ tuma' Wò tùma

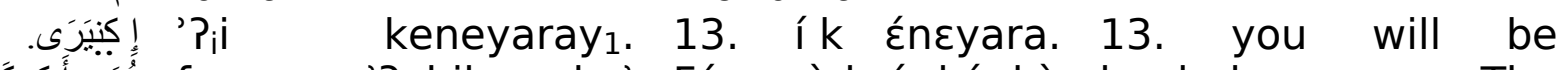

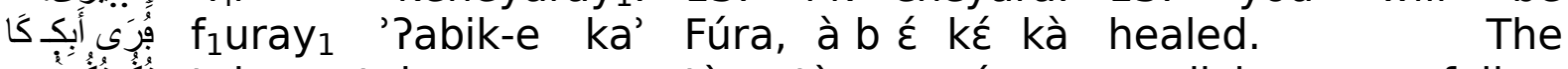

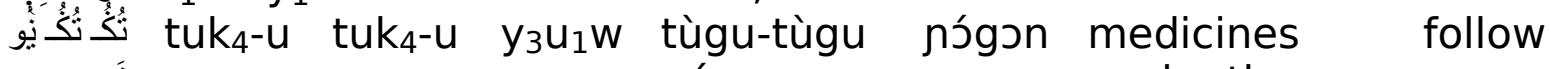

نَ nay 1 ná each other

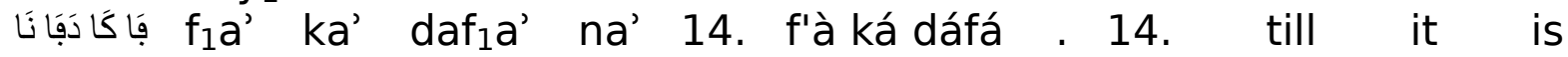

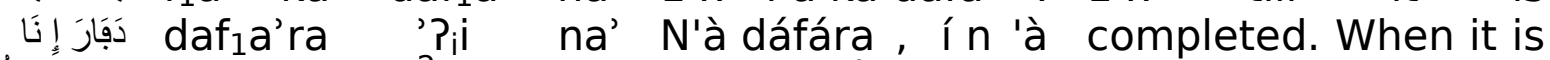

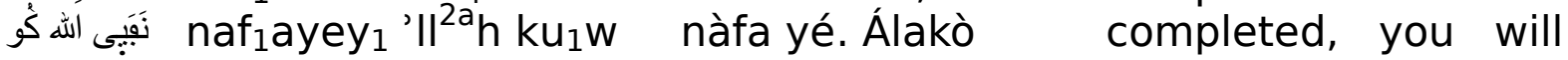
see its benefit. Provided that

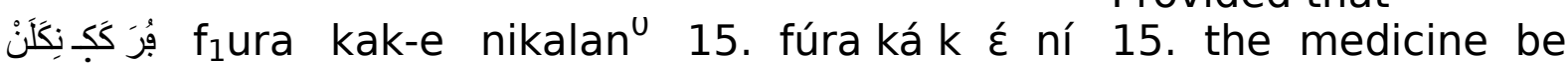

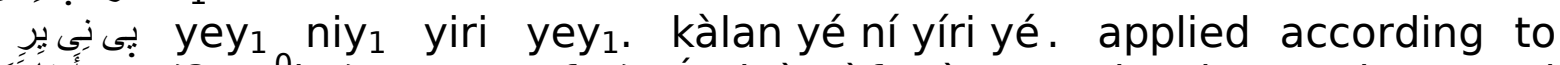
لِّب. أَمْبَا نَبَا in $\mathrm{su}_{1} \mathrm{ru}_{1}$ with a reason. We shall benefit from it

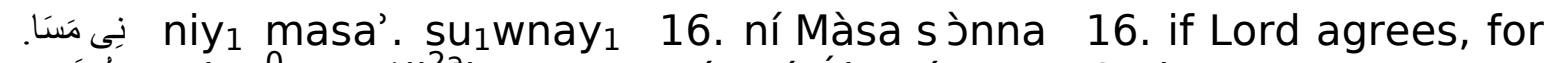

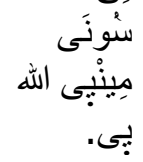
miyn $^{0}$ yey $_{1}{ }^{\prime} I^{2 a} h$ yey $_{1}$. mín yé Ála yé. God.

Text 5

Almamy Yattara's comment:

The possessor of the document "cilissi foura" is again Bory Bary. Found in the belongings of his father Amadou Bary, a Muslim cleric. Copied by Almamy Malik Yattara in 1972 in San. ${ }^{17}$

${ }^{17}$ The original text:

le detantere de dociman de cilissi foura. tougoure Bory Barty tourve danles afer de son pere - Amadou Bary le marabou. copié par Almamy malick yattara a san en 1972 a san 


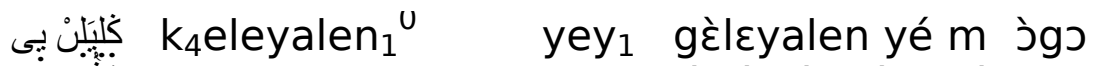

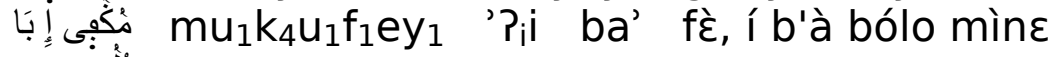
bulu $u_{1}$ mine

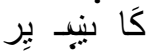

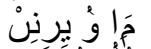

كُخْنَ

ka' у $y_{3} e s-i$ yiri ma' $w u_{1}$ yirinin ${ }_{1}^{0} \mathrm{tu}_{1} \mathrm{k}_{4}-\mathrm{u}_{1} \quad \mathrm{k}-\mathrm{u}_{1}$ kun $^{0}$ ğey $_{1}$

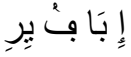

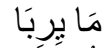

كُنْ

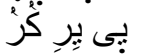

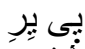

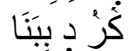

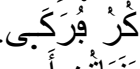

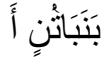

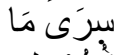

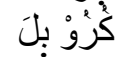

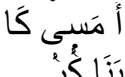

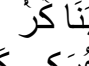

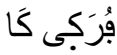

كُنَ

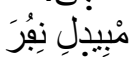

كِّى

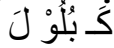

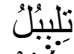

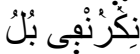

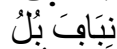

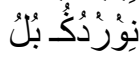

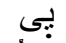

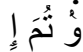

بيجنى

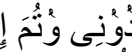

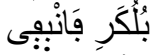

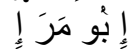

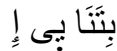

كَّنو إِبَاْ

تُبَ بَنَبَاتُ

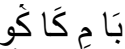

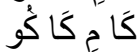

كَا كو فُو

سين土ِ

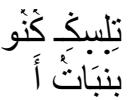

كئيَا.

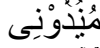

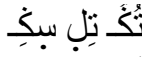

نَا ما كنيا.

'? $\mathrm{i}$ ba' $\mathrm{f}_{1} \mathrm{u}_{\gamma_{2}}$ yiri ma' yiriba' kun ğey ${ }_{1}$ ' ${ }_{j} \mathrm{e}$ de yey ${ }_{1}$ yiri $\mathrm{ku}_{1} \mathrm{ru}_{1}$ yey $_{1}$ yiri

$\mathrm{ku}_{1} \mathrm{ru}_{1}$ de bibana' ku $_{1} \mathrm{ru}_{1} \mathrm{f}_{1}$ urakaey $_{1}$. banaba'tu ${ }^{n}{ }^{n}$ seray $_{1} \mathrm{ma}^{\prime} \mathrm{ku}_{1} \mathrm{ru}_{1} \mathrm{w}^{0}$ bela

'?u masey ${ }_{1}$ ka' bana' $\mathrm{ku}_{1} \mathrm{ru}_{1} \mathrm{f}_{1} \mathrm{urakey}_{1} \mathrm{ka}$ keneya' kun ${ }^{0}$ ǧey.

$\mathrm{m}^{\text {Ubiydeli nif }}{ }_{1} \mathrm{ura}_{1}$ yey $_{1}$ $\mathrm{miyn}_{1}{ }^{0} \quad \mathrm{bi}^{\mathrm{i}} \mathrm{P}_{\mathrm{i}} \mathrm{i} \quad \mathrm{k}-\mathrm{a}$ buluw $^{0}$ la tilebebulu

$n^{n i k u} u_{1} u_{1} n^{u} f_{1} e y_{1}$ niba' $f_{1} a$

niwu $_{1}$ ru $_{1} \mathrm{duk}_{4}-\mathrm{u}$

bulu

bulu

yey 1

${ } \mathrm{wu}_{1}$ tuma ' $\mathrm{p}_{\mathrm{i}} \mathrm{i}$ byğ $\mathrm{u}_{1} \mathrm{y}_{1}$

$d_{1} w_{1} u_{1}$ iy $_{1} \quad w_{1}$ tuma ' $\mathrm{P}_{\mathrm{i}}$ bulukari

$f_{1} a^{\prime} n^{0} b^{2} f_{1} y_{1}$

4. Í b 'à fó yíri mà

yíriba kùnj $\varepsilon$. É dè

yé yíri kòro yé, yíri

5. kذ̀ro dè bé bàna kว̀ro fúraké.

Bànabaato, ní à séra màa kذ̀rıw béc lá

6. ù má sé kà bàna

kว̀ro fúraké k'à kénعya. Kùnje

7. ń b 'í déli ní fúra yé mín b 'í kàn búluw lá tìlebinbulu

8. ní kóronfebulu ní báfanbúlu ní wòrodugubulu yé,

9. wò tùma í b 'í j j̀ dóoni, wò tùma í

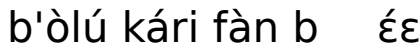
fहे,

' $\mathrm{P}_{\mathrm{i}} \mathrm{i} \mathrm{bu}_{1} \mathrm{w}$ mara ' $\mathrm{P}_{\mathrm{i}} \mathrm{i}$ bitana' yey ${ }_{1}$ ' ${ }_{i} i$ $\mathrm{kasu}_{1} \mathrm{w}$ '? i ba' tu bi banaba'tu

ba' mi ka' ku 1 w ka' mi $\begin{array}{llll}k a^{\prime} & \mathrm{ku}_{1} \mathrm{~W} & \mathrm{ka} & \mathrm{ku}_{1} \mathrm{~W}\end{array}$ $f_{1} u_{1} w$ siysisek ${ }_{4}-i$

\section{tilesek ${ }_{4}-\mathrm{i} \quad k u_{1} \mathrm{nu}_{1} \mathrm{~W}$} bnba'tu ' 'Pa bkeneya'. ' b' muy ${ }_{3} u d u_{1} w^{0}$ niy 1

tuk $_{4}-u$ tile sek $_{4}-i$ na' m' keney'. 'P i bisek 4 -i
10. í b 'ò màra í b ع́ táa n'à yé í ká só , í b'à tóbi, bànabaatว 11. b'à mìn $\mathrm{k}$ 'à kò k'à mìn $k$ 'à kò $k$ 'à kò fóo sìn séegin

12. tìle séegin kóno. Bànabaato, à bé kéneya. À b 'à mùnu dóonin

13. túgun tìle séegin, n'à má the person is seriously ill, take him by the hand

3. and direct him to the plant whose name is kunje (Guiera senegalensis shrub).

4. You say about the plant, kunje big tree. You have seen a big tree, it is an old

5. tree that heals old disease. A sick person, if it was a question of any old person,

6. they could not treat old diseases and heal them. Kunje,

7. I beg you, if all the medicine that you have in foliage, the western foliage

8. and eastern foliage, and northern foliage and southern foliage,

9. then stay a little bit, then cut it everywhere,

10. keep it and bring it to your house, cook it, let the sick person

11. drink it and wash him, let him drink it and wash him, and wash him, up to eight times

12. during eight days. The sick person will recover. Let him wait a little 13. again eight days, if he has not 


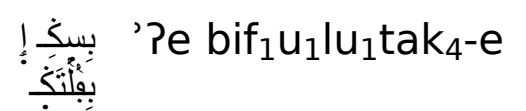

kénعya, í b 'à sègin ,

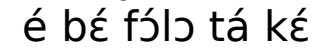

recovered, repeat it, do it as for the first time

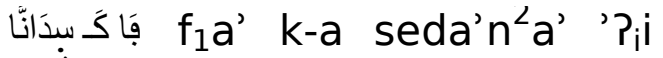

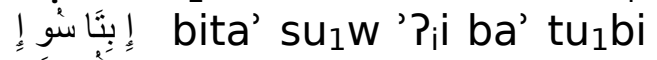

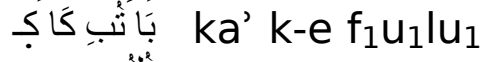

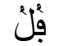

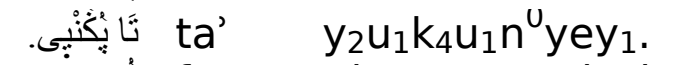

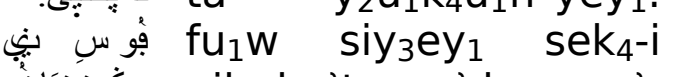

سبذِ نِنبنَّات ' nibnba'tu1 m' keneya'

ما كينيًا

وُ لَونُشَ أ wul la w tuma '? bisa'.

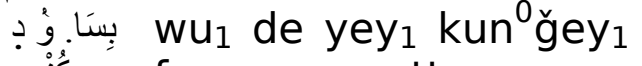

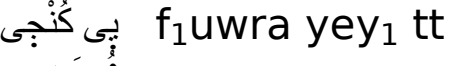

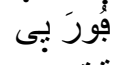

تَتَ

آَبَان a ba’n
14. f'à ká sé dàn ná. Í bé táa só, í b'à tóbi k'à ké fólo

15. tá nògon yé , fóo sì ne séegin. $\mathrm{Ni}$ bànabaats kéncya

16. wò lá, wò tùma à b ć sà. Wò dè yé kùnjzfura yé.

17. À bánna. má 14. till the end. Go home, cook it and do it

15. like the first time, and so, eight times. If the sick person has not recovered

16. this time, then he will die. It is the medicine of kunje.

17. It is the end.

5. Grammatological properties of the Bamana Ajami from San

Table 1: Independent units of the Bamana Ajami orthography Letter Transcription

$1 \varnothing$

ب $\quad b$

$ت \quad t, c$

$\tau h$

r $j$

s d

j $\quad r$

$j \quad j$

w S

ص $\quad S$

ب $\quad f$

ف $g$

s $k$

s $\quad g$

J 1

(18 $m$

18 ن $n$;

$\dot{\varphi} \quad n$

- $h$

, w; length of $u, O, J$

s $\quad y, n$; length of $i, e, \varepsilon$

n $\quad$ n

${ }^{18}$ In final position.
Phonemic value Transliteration

$\varnothing$

/b/

$/ \mathrm{t} /, \mathrm{t}] /$

/h/

/dz/

/d/

$/ \mathrm{r} /$

/dz/

/s/

/s/

/f/

/g/

/k/

/g/

/l/

$/ \mathrm{m} /$

/n/

/n/

$/ \mathrm{h} /$

/W/, N:/

/j/, /n/, /V:/

/n/ b

t

h.

$\dot{g}$

d

$r$

z

$\mathrm{S}$

Ș

$f_{1}$

q

k-

$\mathrm{k}_{4}$

I

$\mathrm{m}$

$\mathrm{n}_{1} / \mathrm{n}$

$y_{3}$

$\mathrm{h}$

w

$y$

$y_{2}$ 
Comments:

- $\tau$ appears only in the name of the Prophet محمد | <mhmd> Muḥammad.

- $\quad j$ is used representing $j$ only once in an Arabic loan, i.e. ${ }^{\prime} \Sigma^{\prime} j \mid<z a k$ '> jáka 'alms' (3:5) (from Arabic zakāt)

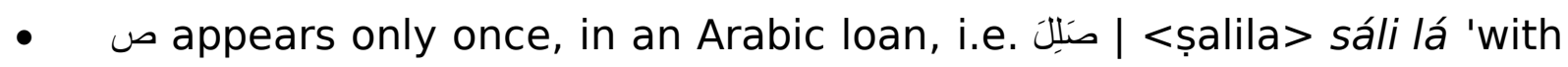
a greeting' (3:1) (from Arabic șalā).

- $\varepsilon$ appears only once (2:15), with its meaning remaining unclear.

- $\quad$ (with two upper dots as in Mašriqī) representing $g$ occurs only once, i.e. in the word (perfective form)' (3:15).

- Nūn in word-final position appears usually without a dot, i.e. as u. However, the dotted variant, i.e. ن, is also encountered in the texts.

- The usual graphemic representation of $n$ as $\div$ (with two lower dots and one upper dot) never appears in word-final positions. If a word has $\Omega$ in the final syllable, $\dot{*}$ is always followed by $s$. Therefore, it is impossible to figure out which Arabic character served as its base, i.e. s or 4 .

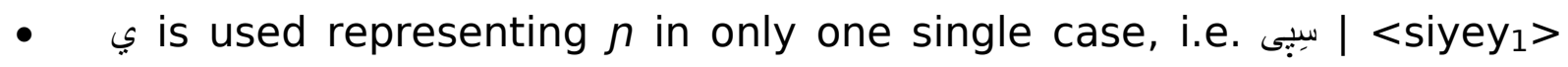
sine 'time' (1/2:6) (which may be a copyist's or author's error).

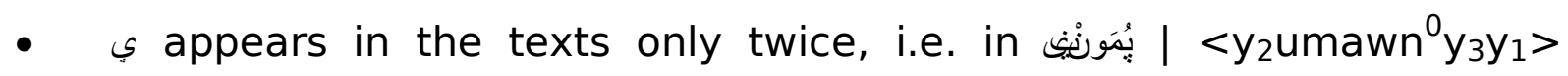

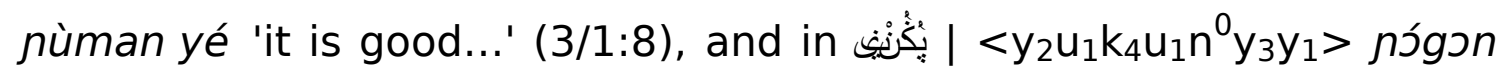
yé 'with each other' (5:15). 
- Prenasalization of initial consonants is marked in the same manner as word-internal nasalization by a nūn with a sukūn, i.e. $\stackrel{\dot{\cup}}{\mid}\left\langle\mathrm{n}^{0}\right\rangle$, such

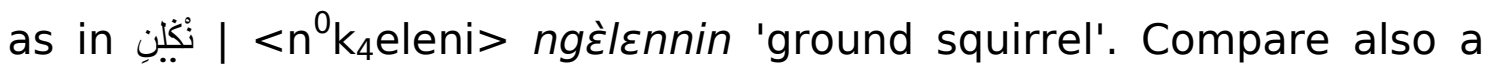
form is attested in Charles Bailleul's dictionary (2007) as ngj̀ (the prenasalization in this word might be absent from the dialect of the manuscript's author).

\subsection{Diacritics}

Fatḥa, kasra, and damma are used for representation of $a, i$, and $u$ respectively, while sukūn represents the lack of any vowel (in fact, it used only with nūn).

Tašdīd is rarely employed representing gemination of a nasal sonant as in e.g. with unclear purpose, e.g. دِمِسَّ |< dimis ${ }^{2} \mathrm{aw}^{0}>$ dénmisenw 'children' (1/1:16). Also, it occurs in Arabic loans in order to reproduce the original

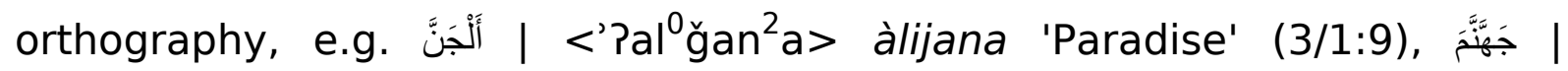
$<$ ğah²an²ama> ${ }^{19}$ jàhanama 'hell' (3/1:9).

In the Bamana Ajami orthography, new diacritics have been introduced for the representation of middle vowels: an 'inversed damma', i.e. : $\mid\left\langle\mathrm{u}_{1}\right\rangle$, representing $o$ or $\mathrm{\nu}$, as well as a lower dot, i.e. $: \mid<e>$, representing $e$ and $\varepsilon$. There is also a combination of lower dot + fatha, i.e. $\because \mid<$ ae $>$, which represents the open front vowel $\varepsilon$, and which is used only

\footnotetext{
${ }^{19}$ The tašdīd on top of the hā' was put here mistakenly by the writer.
} 
in text 4: هَبَر | haera> héra 'be in peace' (4:11), <yerae> yغ̇rع 'self' $(4: 11)$.

Generally, vocalic diacritics are provided. However, they may be omitted from time to time, apparently due to negligence of the writer or copyist.

Diacritics representing vowels which are not preceded by a consonant are placed on top of a supporting 'alif hamza, i.e. $\mid<$ '? or ! | $<' P_{i}>$, such as e.g. in ${ }^{\prime} \mid<' P_{u}>$ ù 'they' $(1 / 2: 6), ! \mid<' P_{i} e>$ é 'you (singular emphatic)' (1/2:3).

Vocalic nasality is marked either by mim in word-internal position, when the nasal vowel is followed by a labial consonant, or by nūn with

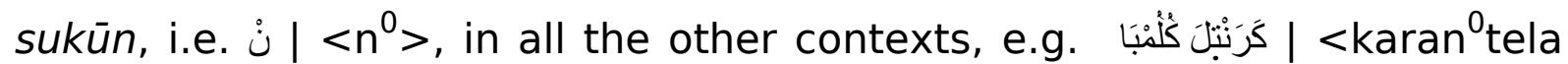

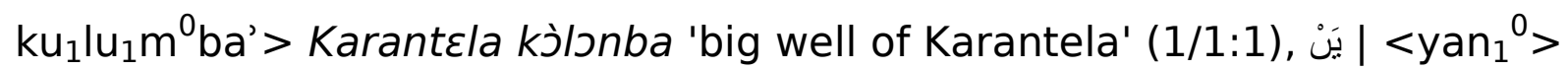
yàn 'here' (1/1:1). Not infrequently, the nasality remains unmarked in word-final positions, such as e.g. $\stackrel{\dot{s}}{\mid} \mid<\mathrm{du}_{1}>$ versus $\dot{\mathrm{j}} \mid<\mathrm{du}_{1} \mathrm{n}_{1}{ }^{0}>$ dón 'to know' (1/2:1) (both spelling variants appear in the same line), بَابَ |

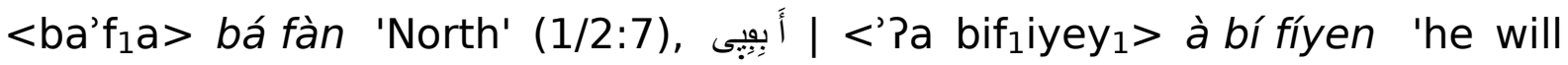
become blind' (1/1:6), كب | k kele> kélen 4:5 'one'. More rarely, and probably by negligence, it also remains unmarked in word-internal

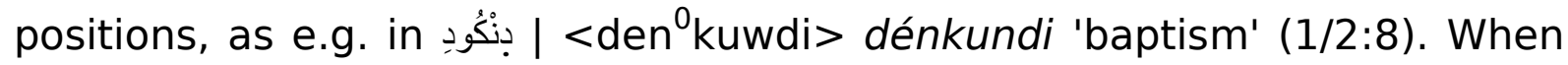
followed by the plural marker - $w$ /ù/, nasality is never marked in the

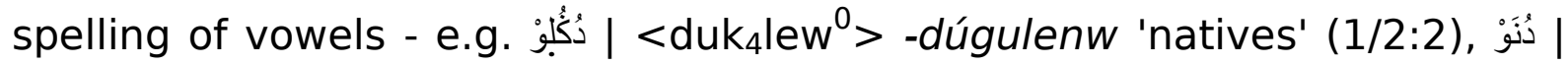
$<$ dunaw $^{0}>$ dúnanw 'strangers' (1/2:1), ندِّ | <nidew ${ }^{0}>$ ní dénw... 'if children...' (1/2:3).

The syllabic nasal ń, which occurs in the first singular pronoun of Bamana is featured in the texts only once, where it is followed by a labial 


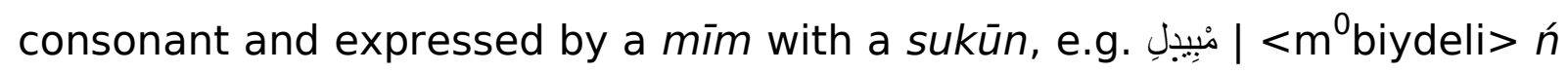
b'í déli 'I beg you' (5:10). Supposedly, in all other contexts it is rendered by nūn (in the same way as prenasalization and syllable-final nasal).

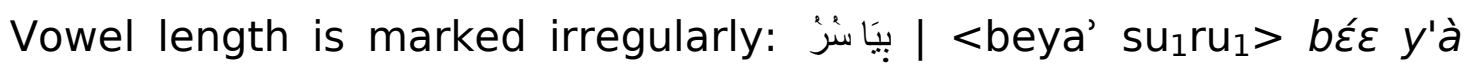

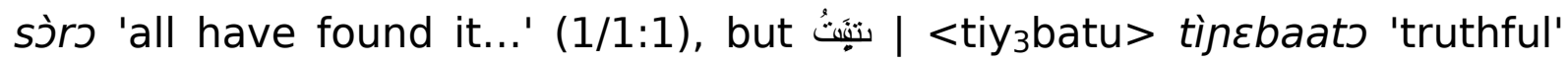
(3/1:3). On the other hand, letters marking vocalic length in Arabic orthography, i.e. 1, , and و, may appear where there is no vocalic length

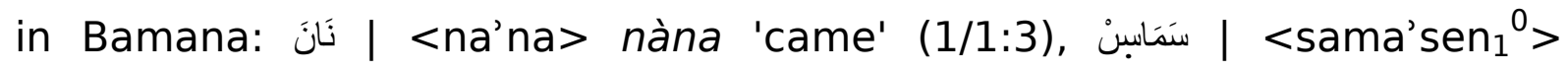
sàmasen 'buttress' (3/1:3), مِين $\mid<\operatorname{miyn}_{1}{ }^{0}>$ mín relativization marker $(3 / 1: 2)$.

Quite regularly, vocalic assimilation (usually referred to in the description of Bamana as "elision") is expressed by a letter representing a long vowel in Arabic orthography, particularly when the resulting vowel is

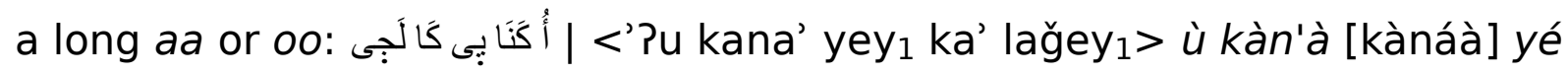

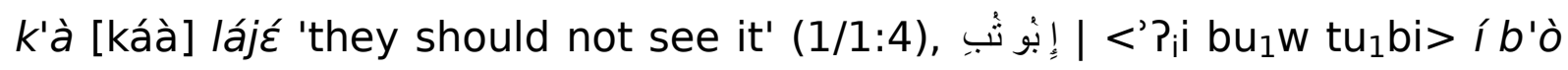
[bóò] tóbi 'you will cook it' (4:5). If this assimilation results in an $i$, it is represented as a separate syllable: In such a case, ii is written as kasra below a supporting 'alif hamza, with an additional kasra on the preceding letter, i.e. $!=\mid<i$ 'P $P_{i} i>$; e.g. wash your feet' $(2: 6)$.

Very frequently, word-finally 'length letters' are encountered which do not express any length in Bamana Ajami orthography, as they do in other orthographic traditions, e.g. that' (1/1:11), سُمَى بيف | < sumay $_{1}$ by $_{3} \mathrm{y}_{1}>$ sùma bí nغ̀ 'food will be abundant'

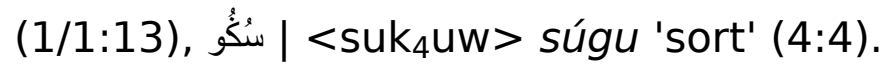


The plural marker, /ù/, is rendered by a wāw with a sukūn, i.e. $\dot{g}$ |

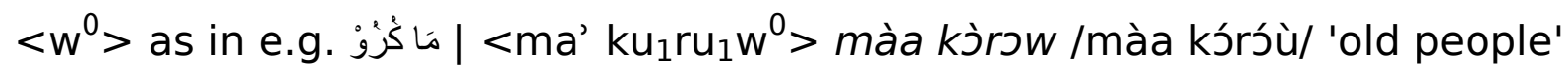
$(1 / 1: 3)$.

The author of the texts makes an effort to maintain the original orthography in Arabic loans, although he does not always succeed, as e.g.

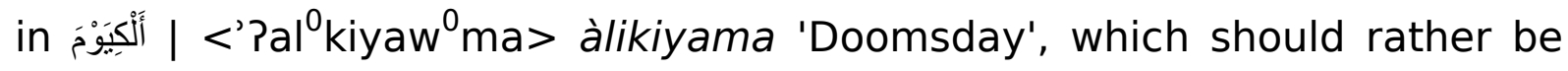
| > القيَّامة > alqiya'mü> al-qiyāma. In any case, the author writes Arabic loans which have undergone phonological changes following Bamana Ajami

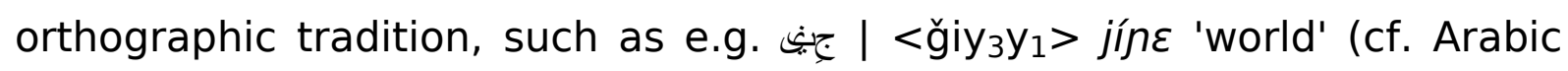
dunyā) (3/1:14),

\subsection{Orthographic segmentation of words}

Segmentation of written speech into graphic words in San Bamana Ajami is more or less orderly (especially in comparison with the specimens published in Vydrine 1998). Content words are normally written separately

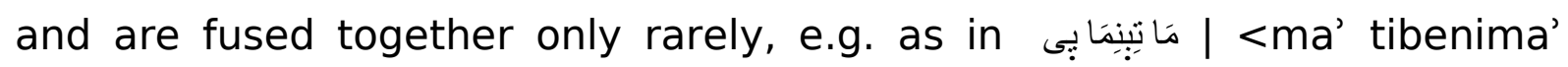
yey $_{1}>$ Màa tź bèn ní màa yé 'people do not meet each other' (literally 'a man does not meet a man') (1/2:9).

Postpositions are mostly, albeit not always, written together with the

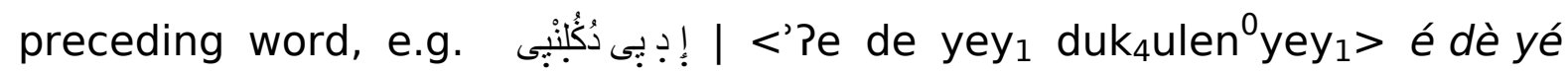

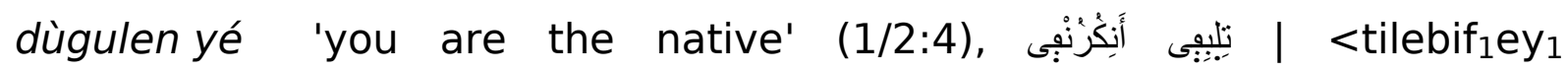
'Paniku $\mathrm{ru}_{1} \mathrm{n}^{0} \mathrm{f}_{1} \mathrm{ey}_{1}>$ tìlebi $f \grave{\varepsilon}$ àní kóron $f \dot{\varepsilon}$ 'on the western side and on the eastern side' (1/2:6). Monosyllabic auxiliaries (predictive markers) are usually written together with either the preceding or the subsequent word. 
In some instances, derived words are split up orthographically in

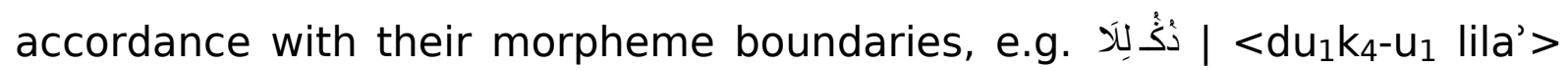
dògoli lá 1/2:9 'secretely'.

\subsection{The dialectal characteristics of the texts}

The manuscripts are written in a Bamana variety quite close to what is today Standard Bamana, with certain features typical of the eastern dialects, such as the imperfective marker bí, as opposed to bé in Standard Bamana.

Most often, the lexeme for 'person' appears in its eastern dialectal form, i.e. Lَ | <ma'> màa (1/1:3,5:5). However, occasionally it may also appear in the western dialectal form, i.e. $\stackrel{1}{s} \mid<\mathrm{mu}_{1} \mathrm{k}_{4}-\mathrm{u}_{1}>\operatorname{mòg}$ (5:1). Throughout all the manuscripts, western forms predominate, e.g. $<$ tu $_{1} \mathrm{k}_{4}-\mathrm{u}_{1}>$ tóg j 'name' (1/1:8, 3/1:1, 5:3 ) (cf. the eastern form twàa).

Occasionally, intervocalic velars are elided, as for example in سِيى |

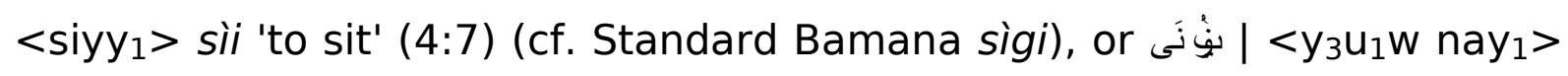
nכ́ón ná 'to each other' (4:13) (cf. Standard Bamana nógวn ná). Elision of intervocalic velar consonants is typical of Maninka and Mandinka languages spoken in the western part of the Manding area, but also for numerous Manding variants on the southern and eastern periphery of that area.

Also, at times archaic forms are encountered in the texts, such as

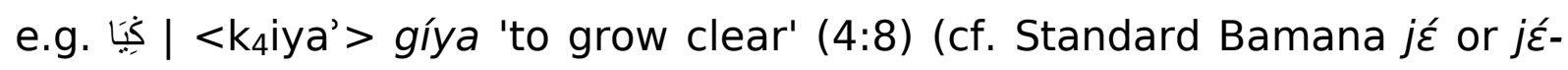
ya, Proto-Manding *xóyi, and in certain Maninka varieties gé). 
References

Bailleul, Charles (2007). Dictionnaire Bambara-Français. 3e édition corrigée. Bamako: Donniya.

Creissels, Denis, Sidia Jatta \& Kalifa Jobarteh (1982). "Lexique MandinkaFrançais". Mandenkan 3:1-207.

Vydrin, Valentin (1998). "Sur l'ecriture mandingue et mande an caracteres arabes (mandinka, bambara, soussou, mogofin)". Mandenkan 33: 1-87. Vydrin, Valentin (2008). "To be respectful in Mande - Where does Maninka honorific vocabulary come from?", Mande Mansa: Essays in honor of David C. Conrad, ed. by Stephen Belcher, Jan Jansen \& Mohamed N'Daou, 216225. Zürich, Berlin: Lit.

Yattara, Almamy Maliki \& Bernard Salvaing (2000). Almamy, une jeunesse sur les rives du fleuve Niger. Brinon sur Sauldre: Grandvaux.

Yattara, Almamy Maliki \& Bernard Salvaing (2003). Almamy, l'âge d'homme d'un letter malien. Brinon sur Sauldre: Grandvaux. 